\title{
Bibliotecas infantiles y juveniles aportación a la política bibliotecaria de Vasconcelos, de Juana Manrique de L ara, primera bibliotecaria mexicana
}

\author{
Martha AliciaAñorve Guillén \\ CentroUniversitariodeInvestigacionesBibliotecológicas \\ de la UNAM, 04510, México D.F.,Tel:56-23- 03- 29 \\ E-Mail:anorve@servidor.unam.mx
}

\begin{abstract}
RESUMEN
El presenteartículo se pro po neubicarlasaportacio nes deJuanaMan ri quedeLara, primerabiblio tecariamexicanacon educaciónformalen bi blio teco no mía, en lapo líticabiblio tecariadeJoséV ascon celosyconello hacerle un lu garen lahisto riadel desarro llo biblio tecario denuestro país de principios del siglo pasado, a las contribuciones de estas figuras del medio bibliotecario.El propósito expresadoconduce a vincular, a partir delasaportacio nesdeMan riquedeLara,losavan cesbiblio teco nó micos lo gradosen elperio do deV ascon celosduran teelgo bierno deCarranza. O tro objetivo deesteartículo espo nerdemanifiesto lavigen ciadelproblemadeaten ción biblio tecariaen relación conlosniñosyjó venes, señalado por Manrique de Lara a Vasconcelos desde octubre de 1921. Con ello setraeal terreno delahisto riadelabiblio teco lo gíaun hecho poco cono cido:quefueunabiblio tecariaquien, en el perio do deAlvaro O bregón conVasconcelosalacabezadelaSecretaríadeEducaciónPública(SEP), llamó laaten ción so brelanecesi dad dequeen lapo líticadedesarro llo bi blio tecario secontemplaralacreación deserviciosbiblio tecariosespecialesparaniñosyjó venes, en tan to queesapo blación represen taríalasemi llaparaformarunaso ciedad lec to ra queusaríalabiblio tecaalo largo de toda la vida.

Parafundamentarsupro puestaManriquedeLaramen cio nólosbeneficios quelereportaríalafundación debiblio tecasinfan tilesyjuvenilesalaso cie dad mexicanay sostuvo quelo menosquepo díahacerseserían sec cio nes paraaten deradichapo blaciónen lasbiblio tecaspúblicaspo pularesquese fun daran. Con estepro pó si to Juanapresen tó un pro yecto paralacreación de estos servicios, señaló estrategias pedagógicas para que los profesores propiciaran el uso de la biblioteca, planteó el perfildeunbiblio tecario infantil, subrayó la importancia de la relación maestro-bibliotecario, y puso de manifiesto la pobreza de literatura de calidad, en español o traducida a este idioma, con la cual atender a dicha población.

Key Words: Biblio te cas In fan ti les y Ju ve ni les, Jua na Man ri que de Lara, PolíticaBiblioteca ria de Ca rran za, PolíticaBiblio te ca ria de Vas concelos
\end{abstract}

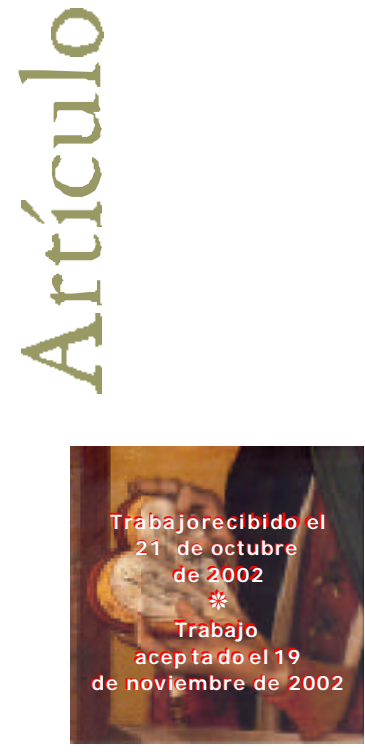




\title{
INFANTI LE AND JU VE NI LE LI BRARIES, ACONTRI BUTION OFJUANA \\ MANRIQUEDE LARA, FIRST MEXICAN LIBRARIAN, TO VASCONCELOS'SLIBRARIAN POLICY
}

\begin{abstract}
MARTha Alicia AÑoRVE-Guillén
ABSTRACT

Thepaperin tendsto lo catethecon tributions of JuanaMan riquedeLara, first formal Mexican librarian, within the library policy of José V asconcelos and thereforegive herthe placeshe deserves in the history of thelibrary devel opment of our country in the last century. This purpose leads to relate Manrique's con tributionswith thelibraryad vancesachieved duringV ascon celos's period in Carran za's gov ern ment. Italso aimsto reveal that theprob lem of li brary atten tion to chil dren and young sters, pointed out by Man ri que de Lara to Vas con celos since O c to ber1921, wasstill in force. This brings to librarian history grounds a fact which is little known: that it was a woman librarian, within theperiod of Presi dentÁlvaro O b regón which had JoséV as co celosas head of the PublicEducation Ministry(SEP), who called the attentionabout the need that the librarydevel op ment policyshould con siderthecreation of special library services forchil dren and young sters, for such popu lation was the seed to con form a read ing so ci ety which would use theli brary for therest of its life. In orderto up hold herpro posal Juanamen tioned the benefits that the foun dation of in fan tileand ju venileli brarieswould bring to the Mexican society, and maintained that the least that could be done was to create sections to attend to such population in the public libraries founded in the future. With that purposein mind, Juana Man rique de Lara designed a proj ect for the creation of such services and pointed out pedagogical strategies for those teach ers who would be in charge of pro pi ti at ing the use of the li brary, setthepro file of an in fan tile librarian, un derlined theim portance of therela tionship teacher-librarian and made evident the insufficiency regarding the quality of the literature existing in Span ish, or trans lated into Span ish, to attend such population.

Key Words: In fan ti le and Ju ve ni le li bra ries, Jua na Man ri que de Lara, Ca rran za's li bra rian policy, Vas concelos librarian policy.
\end{abstract}

\section{INTRODUCCIÓN}

F l presente artículo se propone ubicar las aportaciones de Juana Manrique de Lara, primera bibliotecaria mexicana con estudios escolarizados en esta especialidad, tan to en México como en elex tranjero, den tro delapo líticabiblio tecaria de Vasconcelos y con ello darle un lugar en la historia del desarrollo bibliotecario de nuestro país deprin cipiosdel siglo pasado alascon tribu cio nesdeestafigu radelme dio bibliotecario.

Toda vez que México no halo grado con so lidarunaso ciedadlec to rayqueno ha atendidodebidamentelaimplementacióndeserviciosbibliotecariosparaniños, otro 


\section{Investigacón Biblictecoógia v. 16 N o. 33 julio/ diciembre de 2002}

objetivo de este artículo es señalar la vigencia que aún tiene el planteamiento que Man riquedeLaralemanifestaraaV ascon celosdesdeoctubrede1921 so brelaatenciónbibliotecariaajóvenes.

An tesdeen trarenmateriaconsidero pertinenteseñalarquemiinterésporelestudio delasaportacio nesdeMan riquedeLaradevino delaubicación queMo rales hizo delaim portan ciaqueteníalacon tribución deesteperso najealaen señan zayalaliteratura bibliotecológica mexicana, ${ }^{1}$ y fue precisamenteel estudio detallado de la primera producción escrita de Manrique de Lara el punto de partida que me permitió ubicarsu con tribu ciónalapo líticabiblio tecariadeVascon celosen materiadeservicios bibliotecarios para la niñez y la juventud mexicana, piedra an gulardelaforma ciónlectora.

D esde el punto devistabiblio tecario, elperio do másreco no cido yestudiado dela revo lu ción eseldela "reconstrucción nacio nal" deÁlvaro O bregón, ylafiguradeesa época que en materia cultural y educativa resalta, casi con exclusividad, es la de José Vasconcelos, quienmerecidamentehapasado alahisto riacomo elideólogoyelimpulsor de la educación, laculturayeldesarro llo biblio tecario del México revolucionario. En el medio bibliotecológico es ampliamente reconocido el desarrollo bibliotecario impulsado por Vasconcelos, pero poco seco no cen lasaportacio nesqueMan riquede Larahicieraaestedesarro lloycómo éstaspermearon dicho perio do conlosavan cesal canzados en materia bibliotecaria, que planteaban los intelectuales del constitucionalismoylosbiblio tecariosdelmo men to.Losplan teamien tosdelcarran cismo en mate ria de libros y bibliotecas formaron parte del bagaje que Manrique de Lara y otros perso najesrelacio nadosconloslibrosylosbiblio tecariosdeesaépo callevarondeuna uotraformaaldesarrollobibliotecarioimpulsadoporVasconcelos.

Laubicación yelanálisis delasaportacio nesdeMan riquedeLaraalapo líticabi blio tecariadeV ascon celos obligan are to maral gu nasideasypartes del pro yec to bi bliotecario del carran cismo, queno obstan teserdegran im portan ciaparaelmedio bibliotecario y para la sociedad no eran consideradas, o al menos no manifestaban esto demaneraex plícita, en el proyecto biblio tecario inicialdelaSecretaríadeE ducaciónPública,ejem plo delo cualeslacreación debiblio tecasparaniñosyjóvenes.

D urante el carran cismoJuanaMan riquedeLarahabíato mado plenaconcien cia delbeneficio querepresen tabaparalaconformación deunaso ciedadlecto raylacalidad de la educación, el funcionamiento de bibliotecas escolares o secciones adhoc paralosniñosyjó venesmexicanos. Pero lo cierto esquedes desu con viven ciatemprana y juvenil con el libro y la biblioteca, Juana guardaba una experiencia que más tarde, gracias al contacto formal e informal con el ambiente cultural y bibliotecario orien tado hacialasmayo rías- queeraalo queas piraban losin telec tualesdel carrancismo- , setornó en unafirmecon vic ción porlaqueeraim portan telu char, más aún cuan do el proyec to biblio tecario inicial deV ascon celosno contem plabalacreación

1 EstelaMoralesCampos,EducadónbibiotecolóġcaenMéxico1915-1954, 1988,pp. 8-9, 58-59, 88-89. 
deserviciosbiblio tecariospen sadosen función delascaracterísticas delapo blación infantilyjuvenildelpaís.

ManriquedeLaraconsiderabaquelacreaciónylaoperaciónidó neadeestosespa ciosimplicabaunbeneficio innegableparaelavan cehaciaunaso ciedadlecto raqueel México nacido delarevolu ción san grien tayen plenarevo lu ción edu cativaycultural requería.

Enestatesitura, elpresenteartículo sepro po nereco no cerleunlugaralafigurade Manrique de Lara en la política bibliotecaria de Vasconcelos y conectar, a partir de susaportacio nes, el perio do deCarran zacon aquel otro quesehareco no ci do como la "épo cadeoro delas biblio tecas", 2 no obstan tequeyades deel go bierno deAdolfo de la Huerta el propio Vasconcelos negara los avances que en materia cultural hubierapo dido lo grarel go bierno deCarran zaysedeslin daradedichapolítica, cuan do señalaba: "Lo que yo debo deciresquenuestrasinstitu cio nesdecul tu raseen cuentran todavía en el periodo simiesco de la sola imitación sin objeto, puesto que, sin consultarnuestrasnecesidades, lasorganizan como piezasdeun muestrario paraque elextranjero seengañemirándolasyno paraquesirvan."3

\section{LA ATEN CIÓN BIBLIOTECARIA A N IÑ OS Y JÓVENES DENTRO DEL PROYECTO BIBLIOTECARIO DEL CARRANCISMO}

El contexto bibliotecario del carrancismo es de obligada ubicación cuando nos proponemos com pren deryvalo rarlasaportacio nesqueMan riquedeLarahicieraa la política bibliotecariadeV asconcelos. Muestro acontinuaciónlosplanteamientos que los intelectuales constitucionalistas, encabezados por Agustín Loera y Chávez, formularan parafo mentarlaformaciónlecto rayeluso delabiblio tecadesdelaedad temprana,ylarelaciónqueguardaban estoselementosconlaescuela.

El proyecto biblio tecario del go bierno constitucio nalistadeCarranzafueimpul sado especial men teporAgustín LoerayChávez, quien con tó con elapoyo delsecre tario deInstrucciónPública,FelixPalaviciniparaponerlo enoperación.

Ademásdereorganizarydesarro llarlasbiblio tecasyarchivosdel paísparabenefi cio delaso ciedadmexicana, el pro yecto con tem plabalaideadedesarro llarbiblio tecases co laresyestrategiasparaligarlasalavidaesco lar, asícomo lafun dación deuna biblio tecainfantilenlaBiblio tecaN acio nal (estaúl timafueel ejedelpro yecto bibliotecario del carrancismo)ylacreación delaE scuelaNacio naldeBiblio tecarios, célula in dispen sableparaal can zarel desarro llo biblio tecario ylaformación deloscuadros depersonalbibliotecario.

2 Lin daSametz deWalerstein,V asconcelosehombreddlibralaépocadearodklasbibliotecas 1991. Es esta autora quien lo denomina así.

3 "D eclaracio nesdelseñorlicen ciado donJoséVascon celosconmo tivo delatomadeposesióndel cargo de rectordelaUniversidad Nacio nal deMéxico".p.8. 


\section{Investigacón Biblictecoógia v. 16 N o. 33 julio/ diciembre de 2002}

Laideadelcarran cismo dedesarro llarbiblio tecasinfantilesyasehabíamanifestado enlasCo misionesCulturalesquehabíanidoaEstadosUnidos(mediantelascualeslos profeso resmexicanosrealizabanestan ciasenesepaísparaobservarsudesarrollo educativo yculturalen general), lo queayu dabaasusten tarel desarro llo culturalmexicano, específicamenteeleducativoyelbibliotecario.

Losin telectualesdel go bierno deCarranzaconsideraban quelaculturaylaeduca ciónalcanzadosporlosciudadanosdelosEstadosUnidoseran el mo tordeldesarrollo quehabíanlo grado.A sí, seseñaló lanecesi dad deob servar "el espíritu quehahecho desarrollarse a la República Americana y que la hace adelantar [...] [ya] que el secreto demu cho delo que es bueno en losEs tadosUnidos, debebuscarseen lainfluenciadelasescuelaspúblicas." 4

Con esaideasepedíaalospro feso resob servarno sólo lo quepo dríamosdeno mi nar como el espacioáulico, sino tam bién lain fraestructuraen queéstedescan sabay delaquesenutríalavidaylacul tu raen laescuela. Asíen lasins truccio nes de ob servaciónparalospro feso resseseñalaba: "Engeneral, prestarán suatenciónal mo biliario esco lar, gabinetesdefísica, labo ratoriosdeQ uímica, Museo deHisto riaNatural, bibliotecasinfantilesyescolares." 5

Eldesarro llo biblio tecario, yden tro deél labiblio tecaparaniños, tenían unaimportan ciainnegableen el proyec to edu cativo ycul tu ral deCarranzaporlo quenombraron como comisionado especial para observarelsistemabiblio tecario aLoeray Chávez, yparato marno tasso brelasbiblio tecasyelegirtex tosparaniñosalospro feso resSoto,NoveloyRebolledo. ${ }^{6}$ Alcomisio nado especialseleindicaba:

Estudiaráustedlasobrasliterariasdedicadasalosniños[...]Prestaráatención a la organizaciónde las bibliotecas para niños, tan to fijas como circulantes y coleccionará los catálogos correspondientes. Se informará usted so brelepro cedimien to oficial seguido porlaadmisión detextosesco lares[...]alterminarsusestudiospresentaráuninformedeto dassuslabo resadjun tan do un pro yecto paralacreacióndebiblio tecasparaniños, así como los medios dequeestaSecretaríadebavalerseparaes timulara nuestrosliteratosaquepro duzcan obrasqueven gan aen riquecernuestra literatura nacional escolar.7

Además de estos observadores de las bibliotecas infantiles cabe señalar el énfasis quelapro feso raMaríaAriasBernal-otradelasmen to rasparticipantesenlaCo misionesCulturalesaEstadosUnidos- lesconcedió alosdepartamentosparaniñosdentro de las bibliotecas públicas de Nueva Y ork y que se pone de manifiesto en el informe

4 "ElSeñorD on Venustiano Carran zaesno so lamen teel amigo del pueblo, sino, en particular, de los niños de la escuela de México", p. 102.

5 "Jira[sic]cul tural demaestrosporal gunosdelosCen trosesco lares másim portan tesde los Esta dos Unidos", p. 72.

6 "ElSeñorD onVenustiano Carranzaesno so lamenteelamigo del pueblo, sino en particu lar delos niños de la escueladeMéxico", pp.107-108.

7 Guadalupe Quintana Pali, LasbibliotecaspúblicasenMéxic1910-1940,1988. p. 68. 
que sobre las bibliotecas públicas presentó a la superioridad. ${ }^{8}$ Suponemos que este informefueunso porteim portan teparael pro yec to biblio tecario deCarranzayque también fue una lectura que, entre otras, inspiró las ideas que Manrique de Lara le presentóaVasconcelos.

Seguramenteelresultado deestasaccionesyelgenio deLoerayChávezfuelo que impulsólastressiguientescosas:

a) El desarrollo de bibliotecas en las escuelas primarias superiores "para complemento de la enseñanza" ${ }^{9}$ y el desarro llo delacon ductalecto ra. Espo siblesoste nerquetuvieron ex plícitamen teestaúl timain ten ción todavez quesedijo quese instituían "con el fin esencial de obrando en combinación con la escuela, se fomente en los niños el hábito del estudio y el amor al libro." 10

b) Las estrategiasparaapoyarelanálisisylacom pren sión delalecturayelen riquecimien to edu cativo. Así, seseñaló: "los alum nosque hagan uso deloslibros, al entregar la obra que se les facilite acompañarán algunos breves apuntes en que consten lasideasculminan tes, científicas, mo rales, etc., quead quirieronalleerla; el número de vocablos castellanos desconocidos para ellos, aprendidos durante la lectura por medio decon sul tahechaen elD ic cio nario; y todo giro delen guaje queno hubiesen enten dido paraqueéstelesseaex plicado porlosmaestros." 11

c) Ladeci sión dein cluiren lospro gramas deLen guaNacio nal el temaso bremanejo de bibliotecas y uso, selección y prácticas de asimilación de libros, no solamente enlasescuelasprimariassino también enlasnormales, preparato rias, secundarias e industriales.Parahaceroperativaestaacción LoerayChávez señalaba: "LaEs cuela de Bibliotecarios y Archiveros proporcionará, [...] un programa detallado paraesteapren dizajequereúnalascondicionesdebrevedadyprácticaaplicación a los diferentes grados de enseñanza, iniciando un adelanto que principia con el conocimiento del alfabeto ytienecomo co ro namien to lacul turasu periordeles teta 0 del pensador." 12

d) La implantación de la Biblioteca circulante infantil en el espacio de la Biblioteca Nacio nal.D ado el carácterdeestaúltimaseentien dequeseinstituíacomo biblioteca infantil al servicio de la nación.

8 MariaAriasBernal, "Lasbiblio tecaspúblicasdeEstadosUnidos:informepresentadoal Sub secre tario deIns truc ción Pú blicay Bellas Artes, en marzo de 1915", p.109.

9 "Labor del Ciudadano D. Venustiano Carranza, encargado del Poder Ejecutivo del Gobierno ConstitucionalistadeMéxico, enlaSecretaríadeInstrucción PúblicayBellasArtes, du ran telages tión del Ingeniero Félix F. Palavicini”, p. 225.

10 "Institución de bibliotecas infantiles en las escuelas primariassuperiores", pp. 33-34.

11 Ibid, pp. 33-34.

12 AHBN,FondodelaEscuelaNacionaldeBibliotecariosyArchivistas.Carpeta114.Exp.3360. No. 4354. [Sugeren ciadel directordelaE scuelaNacio nal deBiblio tecariosyArchivistasal Ministerio deEdu cación, dein cluiren lospro gramas de Len guaNacio nal el temadel manejo debiblio tecasy uso, selec ción y prác ti cas deasi miliza ción deli bros. 20 deabril de 1916]. 


\section{Investigacón Biblictecoógia v. 16 N o. 33 julio/ diciembre de 2002}

Laatmósferabibliotecaria, educativayculturaldelcarrancismo,expresióndelinicio deunarevolución culturalquedeseabaim plicarypermearalasmayo ríasenarbo lan do el estandarte de la cultura y teniendo como arma al libro y a la biblioteca,inflamaba a Man riquedeLara, particularmen telalu chaenfavordelaformacióntem pranadelecto resyusuariosdelabiblio teca, lain tegración delabiblio tecayel libro en losdiferentes niveleseducativosesco larizados, yeltemadel manejo debiblio tecas, asícomo eluso, la selecciónylasprácticasdeasimilacióndelibros.

La vida in telectualyemo cio nal deManriquedeLaraseim pregnabadel con tex to cultural quelaro deaba, so bretodo enlaescueladebiblio tecariosyarchivistasyen la Biblioteca Nacional, espacio al que se incorporaría a laborar apenasterminadossus estu diosdebiblio teco no míaen 1917, precisamentecomojefadelacitadabiblio teca circulante infantil. ${ }^{13}$ Con si dero quefue ele gi da como jefadeestabiblio tecano sólo porsusestu diosen biblio teco no míasino también porsupreparacióndenormalista, pues ya para en ton ces había cursado tresaños deesacarreraen el Institu to Normal MetodistadelaciudaddePuebladelosÁngeles. ${ }^{14}$

Enlabiblio tecainfantil, Man riquedeLaratuvo laoportunidad detrabajarcuida do samenteunproyectoconcreto deidentificaciónyevaluacióndeliteraturainfantil y juvenilen español. ${ }^{15}$ Consideramos que si bien este trabajo era útil para la conformación delaco lección delabiblio tecain fan tilacargo deMan riquedeLara, pudo ha berrespon dido alapetición queen viaraLeo no rePower, biblio tecariainfantildeEstados Unidos, a la E scueladeBiblio tecariosso licitan do, antelaescasez deobrasen españolparalapo blacióninfantil, quelosalum nosdelacitadaEscuelaiden tificaran este tipo de literatura. Power necesitaba esta orientación porque estas obras tenían unaaltademandaensubiblio teca:

Con motivo del no tableincremento [...]en elnúmero deestablecimientos [en Estados Unidos] dedicados a la enseñanza de la Lengua Castellana, [que provoca que] multitud deniñosacu den aestabiblio tecaen so licitud de cuentos de leyendas, obras de viaje, etc. escritas en ese idioma. ${ }^{16}$

Tal parece que la selección de obras infantiles y juveniles se le encomendó a ManriquedeLaramientrasocupabalajefaturadelaBiblio tecaInfantil,experiencia quelepermitió aden trarseaúnmásen lapro blemáticaquero deabalaimplan tación

13 AHBN,FondoAdministrativo.Carpeta77.Exp.1908.No.2870.[Relación deoficiosemitidospor la Biblioteca Nacional durante el año de 1917].

14 AHSEP, Fondo de Concentración de la Dirección General de Administración Escolar. Exp. D/ 131/ 4949. D ocumentos de Juana Manrique de Lara. [Copia del certificado que hace constar quelaseño ritaJuanaManriquecursó estu dios, en tre1911y1913en el Institu to NormalMeto dista, Expedido en Puebla de Zaragoza, a los seis días del mes de junio de 1935].

15 Juana Manrique de Lara, "Bibliotecas infantiles y juveniles", p.15.

16 AHBN,Fondo delaEscuelaNacionaldeBiblio tecariosyArchiveros.Carpeta115.Exp.3488. No. 4490. [O ficio turnado porLuis Manuel Rojas, al Sub directordelaBiblio tecaNacio nalyencargado delaEscuelaNacio naldeBiblio tecariosyArchiverosso licitán do leseelabo reunalis ta de obras en españolconvenien tesparaniñosmeno resde15añosparasatisfacerlapeticióndelaSrita.Leonore Power, Chil dren's Li brarian. 9 de agos to de 1917]. 
de servicios bibliotecarios para niños, específicamentela relativaalescaso monto y calidaddelaliteraturainfantilescritaenespañolo traducidaaél.Estosproblemas de terminaron que en su momento Juana identificara la existencia de sólo trescientas obrasdignasdeformarpartedelascoleccionesdebiblio tecasinfantiles.Manriqued e Laracomentaba:

[...]tenemos que conformarnos con unos cuantos [libros para niños] de autores españoles o mexicanos de no mucho mérito, y con algunas traducciones de cuentos para niños de autores extranjeros. ${ }^{17}$

La problemática que existía para conformar colecciones infantiles y juveniles de calidadfuereto madapor Man riquedeLaraen el pro yecto parael diseño debiblio te casdeestaín do lequeofrecióalasauto ridadesdelaSEPaprin cipiosde1922.18

Eltrabajo deselección deestasobrasfuehecho porJuanaconelmayorpro fesio na lismo. Co men tó quelas obrasqueeli gió paraconstituirlalistadecercadetrescien tas obras "adecuadasalasmen tesinfantilesyjuveniles" fueron en primerlu garubicadas en fuen tesau to rizadasyqueen mu chos casoslas obrasmismasfueron leí das porella paravalorarquereunieran "trescondicionesfundamentalesenunlibro desuíndole: $1^{\text {a }}$ quetuvieran un temasu gestivo al al can cedelasjóvenesinteligen cias; $2^{2}$-quefueran de unaintachablemoralidady3 ${ }^{a}$ quesusauto restuvieranfamadebuenosescrito res." 19

D eestaformatan to losco no cimientosdebiblio teco no míacomolospedagógicosy laslecturasyviven cias deMan riquedeLaracomojefadelaBiblio tecaInfan til,lepermitieron elabo rarun nuevo pro nun ciamien to, a prin cipiosde1922, afavordequeel go bierno diseñaraeimplantaraverdaderasbiblio tecasparaniñosyjóvenes, unproyecto mo delo paralaimplantacióndeloqueelladenominóbibliotecainfantilmexicana. ${ }^{20}$

\section{PRIMEROSPLAN TEAMIEN TOS Y PRO YEC TOSEN FA VOR DE LACREA CIÓN DE ESPACIOSBI BLIO TE CARIOSPARANIÑ OSY JÓ VE NES, QUE OFRE CIÓ JUAN AMAN RI QUE DE LARAALAPO LÍTICABIBLIO TE CARIADE VASCONCELOS}

Para1921, tiem po en queMan riquedeLaraleformularaalareciénfun dadaSecre taría de Educación Públicael llamado deaten ción so brelanecesi dad decrearespa cios bibliotecarios idóneos para niños y jóvenes como acciones especiales para cimentar la conducta lectora y el uso de la biblioteca, así como recursos para

17 Man riquedeLara, p.15.Porcierto lafal tadeliteraturaescritaen españolparanños puederelacionarse con la fal tadedesarro llo biblio tecario paraesesectordelapo blación, ob servado tanto en México como enEspaña.En 1918o 1919laseño ritaBeatrizG alindo señalabaqueapesardel desa rro llo delaBiblio tecaNacio nal nadiese habíaacordado delosniños, porlo queen tendemosque en ese país tampoco existían biblioteca in fan tiles, porlo menos hastalos añoscitados, y AHBN, Fondo Administrativo.Carpeta88.Exp.2355.No.3324. [Recortedelartículo "BibliotecasInfantiles" por BeatrizG alin do, publicado en el perió dico El Sd de Madrid. Este artículo fue enviado porel Rec toralaBiblio tecaNacio nal, 28 deenero de 1919].

18 Man ri que de Lara, pp. 15; 18-19.

19 Ihid, p. 15.

20 Ibid 


\section{Investigacón Biblictecoógia v. 16 N o. 33 julio/ diciembre de 2002}

do cumen tarse, ins truirseyrecrearsealo largo delavida, hacíayacuatro añosqueha bíaegresado delaEscuelaNacio naldeBiblio tecariosy Archiverosyhabíasido nombrada como responsable de la biblioteca in fan tildelaBiblio tecaNacio nal. En el momen to en queescribealaauto ridadesdela SEP, labo rabacomo em pleadatécnicadela Nacional, puesto desdeelqueseo cupabadeseguirel derro tero biblio tecario delpaís.

Lospro nun ciamien tosdelaau to raenfavordelainstau ración debiblio tecas o seccio nesparaniñosyjóvenessepublicaron enBiblos, Bo letínInformativo delaBiblio te caNacional[...]"consagradoalavulgarizacióndeloscono cimien tosbibliográficos [...] y [...] de todo aquello que despierte el interés y la curiosidad en cuanto tenga relación con las ciencias bibliográficas." 21 Esto reflejaquedadalaespecialidaddelBoletín,los plan teamien tosypro puestashechaspor Man riquedeLaradebieron habersido co nocidas por las autoridades del movimiento bibliotecario. Estos son los artículos que contienenlaspropuestas:

1).- "Labiblio tecapúblicaylosalum nosdelasescuelaspreparato rias", en Biblos III, 145 (29 octubre, 1921), p.175. (Anexo1.)

2).- "Las bibliotecas públicas y los alumnos de las escuelas primarias" en Biblos III, 146 (5 noviembre, 1921), p. 179.22 (Anexo2.)

3).- "Bibliotecas infantiles y juveniles", en Biblos IV , 158, 159 (28 enero, 4 febrero, 1922), pp. 15; 18-19. 23 (Anexo3)

Como yasehaseñalado, paraelmo mento en quelapren sacapitalinainformaque larecién creadaSecretaríadeE ducación Pública-SE P- fun daráenfechaspróximas lasdiezprimerasbiblio tecaspúblicaspo pularesenlacapital del país,Juanaseperfila ya como una pro fesio nal delabiblio teco no míacom pro metidaconel desarro llo bi bliotecario nacional y la política bi blio te caria del país. Esto es asíal grado de que en medio de la alegríayel reco no cimien to quelesignificaralaim plan tación deesasbiblio tecas, sedetieneafelicitaralaSE Pyal pueblo mexicano pordicho acierto alavez queseñalayproponeaccio nesparaaten derunaausen ciaim portanteenlaformación delecto resydeusuariosdebiblio tecas:laaten ción específicadelapo blaciónin fantil yjuvenil,ademásdepro ponerestrategiaspedagó gicasyadministrativasparaestablecer la relaciónescuela-biblio teca.En dicho con tex to ubicael perfildel biblio tecario infantil, el papel que éste debe desempeñar y la relación que debería haber entre maestroybibliotecario. 24

Tanto en suprimerartículo como en elúl timo delosaquímen cio nadosJuanaubi calaim portan ciaqueparaelpueblo mexicano tienelaim plantación, porprimeravez enMéxico, debiblio tecaspo pulares.Esto eslo queescribeen1921:

21 ["Primer edito rial deBiblos"], p. 1.

22 Aunqueesteartículo seseñalacomo con tinuación delan terior, al habersepublicado con títu lo diferen teal quelean tecedelo heregistrado porseparado. Esteartículo fuepublicado tam bién en la Reista Mexicana deEduracón.

23 Este artículo consta de dos partespublicadas bajo el mismo título.

24 Juan ManriquedeLara, "Lasbiblio tecasinfantilesyjuveniles”, p.15. 
Próximamente, como ya lo ha anunciado la prensa, se establecerán en esta capital diez bibliotecas populares, dependientes de la Secretaría de Instrucción Pública.

Hay quefelicitarnosporlano ti cia, pues bien sabido es queellasven drán a llenarunaurgentenecesidaddenuestro medio,yquesuinfluen ciabenéfica sedejarásentiren elpúblico,yespecialmenteenlajuven tudqueestudia. ${ }^{25}$

Y para1922señala:

¡Escuelas y bibliotecas! Es el grito que nace de todos los corazones que pi den parala patriaun nivel cul tu ral másalto.Y pordon dequierase han establecido nuevas escuelas y nuevas bibliotecas. 26

No obstanteello, reco no celaurgenciadeavanzarenfavordelaconformaciónde una sociedad lectora a través de la formación de los niñosylosjó venes, yen con se cuen ciaen lanecesidad dedestinarespaciosybiblio tecasadhocpara esta población. Asíafirma:

En México no se lee. Los mexicanos no aman la lectura.

Lasbiblio tecasseen cuen tran casivacíasy loslibrerosno hacen losgrandes nego cios que debían ha cer[...] la mayorpar te delos po cosqueleen, pierden su tiempo leyendo libros malos o insulsos [...].

Es, pues, uno de nuestros principales deberes fomentar en nuestros niños el amor por la lectura, pero por la buena lectura, [...].27

Agregaenotrolado:

Laescuelaen nuestro paísdesgraciadamen teno cumplecomo debieraen todos sus aspectos edu cativos. Así, por ejem plo, en lo quesere fiereala educación moral y estética, debemosreco no cerquedejamu cho quede sear.Y aquíesprecisamen tedondelabiblio tecapuededarnosunapo de rosa ayuda como medio de educación. 28

Envirtuddelaimportan ciaquelaau to raleconcedealabiblio tecaenlaeducación, ubicaaésta, además demedio paralaedu cación, como ins trumen to paraen con trar losmediosparainstruirse, poreso señala:

Se dice actualmente [que] la instrucción consiste en saber encontrar los medios de ins truirse(1) [aquí citaLalibraniepubliquedeMo rel]. Hay, pues, que hacerlo comprender al alumno desde lo más temprano posible, y ayu darlo aen con trardichosmedios. Cuan do sehayarecibido yaban do ne parasiem prelaescuela, pro bablemen teten dráquerecurriralasbiblio te cas en cualquier necesidad o duda de su profesión. ${ }^{29}$

25 Juana Manrique de Lara, "La biblioteca pública y los alumnos de las escuelas prepartorias", p. 175.

26 Juana Manrique de Lara, "Bibliotecasinfantiles y juveniles", p.15.

27 Juana Manrique de Lara, "La biblioteca pública y los alumnos de las escuelas prepartorias", p. 175.

28 Ibid

29 Ibid 


\section{Investigacón Biblictecoógia v. 16 N o. 33 julio/ diciembre de 2002}

Al ubi carlaim portan ciadeluso delabiblio tecaen laformación tem pranacomo recurso de ins truc ción alo largo detodalavida, laau to raselamen tadelafal tadebiblio tecasespecialesparaniñosyjóvenesyleseñalaala SEP lanecesidad dedestinaral menosdosdelas biblio tecaspo pu laresqueen 1921 seabrían al pú blico, parauso exclusivo delosalum nosdeescuelasprimariasseñalan do queestásno existen, cosaque reiteraen1922:

Todos los mexicanos podrán en lo sucesivo leer los libros que deseen, gratuita y cómodamente en las bibliotecas publicas que se han fundado en el país.

Pero que daporaten deralosmilesymiles deniñosqueca recen del privi legio de tener una biblioteca especialmente dedicada a ellos.

Lapri merasesu po neque debeser para ni ños has ta de diez años, y lase gunda, para jovencitos desde esa edad hasta los diez y seis. ${ }^{30}$

Ya desde en ton ces(1921)Juanahabíaseñalado quelasbiblio tecasescolaresque existíannopertenecíanensentido estricto aestacatego ría, porello sostenía:

Podría objetárseme que muchas de las escuelas superiores y universitariastienen anexaunabiblio te capara el uso desusalum nos, pero no quie ro tratar aquí de esas biblio tecasrepletasdemanualesylibrosdetex to, a lascualeslamayorpartedelasvecesocu rren losalum nos sólo pornecesi dad, y a veces sin obtener satisfacción completa en los libros que ahí se encuentran. Estas bibliotecas son demasiado especialistas y sólo se encuentran en ellas librosdedo cumen tación o deins truc ciónso bredeterminada rama científica o profesión. Además, su uso se restringe a los alum nos delas es cuelas respec tivas, y casino asisten aellas per so nas extrañas a los planteles. 31

EnlaanteriortesituraMan riquesostienequelasbiblio tecasno sólo son paraapoyar la instrucción, y señala que las biblio tecastienen en realidad tresfines esen cides quepermiten dimensionarsupapeleducativo, deben "[...]instruir, documentarydis traer" 32

Enestatareain tegral delafun ción educado radelabiblio teca,Man riquedeLarasus tentalanecesidaddequeenlasbiblio tecasinfantilesyjuvenilesexistaunbibliotecario com petenteparaaten derlas, al guienformado tanto en biblio teco no míacomo en peda gogía y psico lo gíain fantil,ypo seedordeunavastaculturageneral. ${ }^{33}$ Comprende que esteperfilesdifícildeobtener, pero lo considera"in dispen sablesisedeseaunbuen bibliotecario de niños." 34 En el contexto de la relación escuela-biblioteca, Juana ubica tam bién lademaes tro biblio tecario ycon sideraquesibien el biblio tecario es "elalma

30 Juana Manrique de Lara, "Bibliotecas infantiles y juveniles",p.15.

31 Juana Manrique de Lara, "La biblioteca pública y los alumnos de las escuelas preparato rias”, p. 175.

32 Ibid

33 Juana Manrique de Lara, "Bibliotecas infantiles y juveniles",p.15.

34 Ibid 
deunabiblio teca", 35 ésteyelmaestro "deberán sersiem preco labo rado resmutuosen lamagnaobradelaeducación patria." 36 Asícon si derabaque: "Ensusmanosestáen comendada la educación de la niñez y de la nación en general." 37 Señala que si bien "el maestro debe ser ayudado constantemente por el bibliotecario", a este último "toca, por su parte, cumplir con sus propios deberes, atendiendo con eficacia a sus jóvenes lectores, enseñándoles el correcto manejo de los libros,sugiriéndoles el deseo de leer determinadasobras." 38

Envirtud dequeparaMan riquedeLaralabiblio tecadebeformarenla "buenalectura", "laqueinstruye", "ladeleitaelevan do elalma, porlaquepresen taanuestro es pírituhorizontes más amplios y bellos; porlaquefortificaelalma." ${ }^{39} \mathrm{Y}$ cuando hay una fal tadeculturadelectura, porescasez deobrasin fan tilesyjuvenilesdevalía en español, Man riquelecon cedeaún mayorim portan ciaalaguíaen laselec ción delectura al papel especial que desempeñan el maestro y el bibliotecario en su selección. Respecto de la importancia que tieneel pro fe soral co no cera cadauno de sus alumnoscomenta:

Hay, pues, que poner a su al can ce[delosniñosyjó venes]librosbuenos, librosquepasan do por el examen sabio del maes tro que co no celapsico lo gíaespecial decadauno desusalum nos, puedan sersuminis tradoscon el cuidado que transmiten los conocimientos científicos..$^{40}$

Encuantoalbiblio tecarioinfantilindicaque:

[...]debeserun edu cador, un maestro yun co labo radorefec tivo yvalio so delosmaestrosdelasescuelas, además deunamigo cariño so ein teligen te delosniñosyjó venesquecon cu rran al establecimien to, alos cuales ayu darácon suscon sejosyguiaráen laformación desusgustosliterariosyen la selección de obras de buenos autores. 41

La autora considera que la lectura es formativa y que las primeras lecturas dejan una impronta en el niño, razón por la cual comenta: "El niño no olvidaránuncasus primeraslecturasysuinfluenciasedejarásentirtodasuvida." 42

Respecto delasestrategias paraasistiralabiblio teca, en tan to quelaau to racon sideraque "labiblio tecaesun po dero so auxiliardelaescuelayel tiem po queseem plee en asistir a ellas, ya sea para recreo o para instrucción del alumno, hará más por su educaciónyculturageneral,(delniñoydeljo ven)quesiesemismo tiem posedestinara

35 Ibid

36 Ibid

37 JuanaMan riquedeLara, “Lasbiblio tecaspúblicasylosalum nosdelasescuelasprimarias”, p. 179.

38 Ilid

39 Juana Manrique de Lara, "La biblioteca pública y los alumnos de las escuelas preparđorias", p. 175.

40 Ibid

41 Juana Manrique de Lara, "Bibliotecasinfantiles y juveniles",p.15.

42 Juana Manrique de Lara, "La biblioteca pública y los alumnos de las escuelas prepartorias", p. 175. 


\section{Investigacón Biblictecoógia v. 16 N o. 33 julio/ diciembre de 2002}

paraelestudio decual quiermateriacientífica" .43 Pro po nequesereformen los ho ra riosesco lares, ylosdelasbiblio tecas, detalformaqueéstas "estuvieranabiertasu na 0 másho rasdespuésquelasescuelas." 44

Tambiénpropo neespecíficamenteestrategiasenlasquelabiblio tecaayudeamejo rarlainstrucción es co lar. Así, ofrecealosmaestrosideasparafo men tareluso dela biblio teca,tales como el desarrollo de temas y el estu dio con mayoram plitud delas materiasescolares. 45

En cuanto a la formación del hábito de la lectura la autora considera que el uso obligado delabiblio tecacon tribuiráasuformación, asíco menta: "Elalum no iría[...] porobliga ción al prin ci pio, pero unavez formado el hábi to yel amorporlalec tura, fácilmenteiráporsugusto." 46

Manrique de Lara pone tal confianza en la formación de ese hábito que considera quellegaríaunmo mento en queelalumno preferiríaasistiralabiblio tecaquealcine. ${ }^{47}$

Con toda esta argumentación a favor de la fundación o el destino de algunas biblio tecasescolaresparaeluso delosniñosyjóveneslaauto rapresentafinalmente un proyecto "para el establecimiento de una biblioteca infantil mexicana," 48 toda vez quead viertequepara1922no haybiblio tecasinfan tilesyjuvenilesen México.D ice entonces:

Actualmen teno hayunasolabiblio tecain fan tilojuvenilenlaRepública, ymien trasen otros países haceyalargo tiem po quelosniñostienen ya ese beneficio, en treno so trosmu chosmilesdeelloscarecen deun estableci mien to adon depuedan con curriraleerloslibrosadecuadosasuedad. 49

Paracon tribuirahacerviablelacreación deunabiblio tecainfantilmexicanalaau to rapresentaun proyecto quecon tem plalossiguientesaspectos:a)lo cal,b)mo biliario, c) biblio tecario, d) libros, e) lecto res,f)catalo gaciónyclasificaciónyg)hora del cuento.

En cuan to allo cal pro po nequesea espacio so yquesuam plitud esté "pro porcionada al número de lectores que se suponga habrá de contener." ${ }^{50}$ La autora pone también atención sobre condiciones de buena iluminación diuma y nocturna, de buenaventilación, sostienequehayqueevitarlahumedadyelfrío, esto último porel daño quecau sanaloslibros. Y con tem plareglashigiénicasacordesparacualquieres tablecimientopúblico.51Encuantoaladeco racióndeestetipo debiblio tecasafirma:

43 JuanaMan riquedeLara, “Lasbiblio tecaspúblicasylosalum nosdelasescuelasprimaias”, p. 179.

44 Ilid

45 Ibid

46 Ilid

47 Ihid

48 Juana Manrique de Lara, "Bibliotecas infantiles y juveniles", p.15.

49 Ibid

50 Ibid

51 Ibid 
El salón de lectura de una biblioteca infantilojuvenil, deberáseralegre, con sus pare des adornadas de cuadros con asun tos al al can ce desusvisi tantes, y si es posible tendrán plantas y flores. ${ }^{52}$

Respeto del mobiliario recomienda que sea especial: "Las mesas redondas son preferidas,yéstasylassillasadecuadasalaestaturadelosjóveneslectores." 53

Conrelaciónal sistemadeestan teríaseñalaquedeserabierto serequierenalgunos estan tesbajos; no obstan teco men taqueno reco mien dadicho sistemaporlafal tade formación queexisteen cuan to al uso delabiblio teca: "[...] so lamen tedespuésdealgúntiem po detrabajar[...]enunabiblio tecainfantilconnuestrosniños, po dríaresolversesiesdeimplementarse." 54

En cuan to aloslecto resprecisaqueparaeste tipo debiblio tecashaymilesymiles deniñosquepo drían pro venirespecialmentede "lasescuelas, lasfábricasylasoficinas donde trabajen niños y jóvenes", quienes ten drían queserclasifica dosporgruposdeedad.55Comentaquealbibliotecario esaquiencorresponde:

[...]haceragradableydeseablelavisitaalsalón delectura, mantenerladisciplinamásestricta,yvigilardiscretamenteasusjóveneslectores. [Él]les ayudará a escoger sus libros y procurará enseñarles la mejor manera de tratarlos, protegiendo sus pastas, evitando que se ensucien y volviendo correctamente sus hojas. 56

En cuan to alacatalo gación reco mien daladeMel vilD ewey, apartirdelaobrade JuanB.Iguíniz"Instruccionesrudimentariasparalacatalo gaciónylaclasificación de lasbibliotecaspopulares." 57

Y finalmen teestásureco men dación so brelaim plantación delahoradel cuen to, quedestacacomounaactividadim portanteyacostumbradaenbiblio tecasinfantiles ex tranjerascomo formadedespertarel deseo deleercuen tos dehadas, bio grafíasnotables,leyendas,mitosoargumentosdeobrasclásicas. ${ }^{58}$

El llamado de Juana Manrique de Lara a la política bibliotecaria puede cerrarse, al menosformalmente, con unafrasedeellamismaen laqueparecemanifestarsuexpectativa de que nuestro país contara, como base para avanzar y colocarse entre los más destacados, conun desarrolloparalelo deescuelasybibliotecas, aspiración queincluso enlosalbo resdelsiglo XXI estálejosdeal canzarseennuestro país. Esto escribíaManri quedeLarasiguien do aJu lio Simón: "[...]el pueblo más gran dedelatierraesaquel que tienemayornúmero deescuelas" , alo cualagrega "ytambiéndebiblio tecas." 59

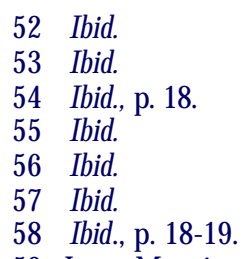

59 JuanaMan riquedeLara, "Lasbiblio tecaspúblicasylosalum nosdelasescuelasprimarias”, p. 179. 


\section{InvestigacónBiblictedoógica v. 16 N o. 33 julio/ diciembre de 2002}

\section{AC CIO NESAFA VOR DE LASBI BLIO TE CASY LALEC T U RAIN FAN TIL YJU- VENIL EN LA POLÍTICA BIBLIOTECARIADE VASCONCELOS}

Lasobservacio nesypro puestasformuladasyofrecidasporManriquedeLaraentreoctubrede1921 yprincipiosde1922alapo líticabiblio tecariadeV asconcelos, se vieron deunau otraformaplasmadas en accio nesllevadasaefec to porlaSEP durante la gestión de Vasconcelos en fechas posteriores a los señalamientos hechos por ManriquedeLara.Citamosacontinuación al gunasdelasaccio nesem pren didaspor Vasconcelosafavordelfo men to delabiblio tecaylalecturaen trelapo blación in fantilyjuvenil:

Para atraer a la población infantil a las bibliotecas populares, en ju nio de 1922 el Reglamen to debiblio tecaspúblicasestablecequelosresponsablesdeestasbiblio tecasllevenaefecto lo siguiente:

Hacer una propaganda real y efectiva para lograr el aumen to delecto res en la Biblioteca, en las fábricas, talleres y escuelas del lugar. ${ }^{60}$

Losen cargados delas Biblio tecas deben pediralos direc to resdelasEs cuelas que existan en ellu gar, quelleven alosalum nosalas salas delec tur raal efec to deacostum brarlosdesdepequeñosafrecuen tarestoscen tros de cultura. ${ }^{61}$

EnlaConferen cialeídaen el "Continen tal Memo rialHall” deWashingtonafina lesde1922, Vascon celoshacepúblico el hecho dequelasbiblio tecaspo pularescontaban con unacolección especialdelibrosinfantiles. ${ }^{62} \mathrm{~A}$ un quelasbiblio tecasinfanti lesyju venilesadhocque Man riquesugeríato davíano habían sido creadas, lo an terior dabarespuestaalaatenciónaestapoblación, almenosconcoleccionesespeciales.

Porotrolado enlaConferen ciaan tescitadaV asconceloshacíaexplícitassusideas acercadelarelaciónescuela-biblio teca,yseñalabaelpodereducadordelabiblioteca:
[...]. La biblio teca-decimosalosmaes tros- es el com plemen to delaes cuela. D espuésdequeseapren dealeer, esnecesario saberlo quedebele erse y disponer de libros. Una buena biblioteca puede substituir a la escuela y aun algunas veces superarla. Una buena biblioteca es una uni- versidad libreyeficaz.Estanim portan tecrearbiblio tecascomo creares- cuelas. Para muchas cosas no hay universidad tan útil como media docena de libros buenos. ${ }^{63}$

No obstante esto, hacia diciembre de 1922 Vasconcelos todavía no mencionaba quesehubiesen creado serviciosbiblio tecariosespecialmenteparaniños, nieditado publicacio nesparaestapo blación. To davíaamediadosde1923, laSEPreco no cíano haberabierto serviciosbibliotecariosespecialesparaniños.

60 "Reglamento de la Bibliotecas públicas",p.26.

61 Ilid

62 José Vasconcelos, “Conferencia leída en el 'ContinentalMemorial Hall' de Washington”, p. 13

63 Ilid, p. 12. 
A síen elartículo "Lasbibliotecaspúblicasen México" publicado enEllibroyd pue bloen agosto y sep tiem brede 1923, en el ren glón dedicado alas bi blio tecas paraniños, laSEP anun ciabalacreación deanexosparaniños en todabiblio tecaquesefundaraenlosucesivo:

Hastahacetresañosno habíaen México unasolabiblio tecain fan til.Los niños, [...] tenían queacu dirasalo nes como losdelaBiblio tecaNacio nal o el delaBiblio tecadel Museo, en don decomo esnatural, hallaban todo, menoslo quedeseaban, esdecir,lecturasfáciles, instructivas, amenas, aptas para enriquecer la imaginación y dar un cauce al ensueño. Com pren dien do estanecesidadurgen te, seharesuel to quetodabiblio te ca que se funde tenga un anexo infantil. 64

Fue hasta finales de 1923 cuando se anunció en "El libroye pudbo", el establecimien to deunabiblio tecapropiamenteinfantil,enlossiguientestérminos:

LaBiblio tecaInfantil[...]laestablecidaporlaSecretaríadeEducación,es un paraíso paralosniñosquevan a so ñarahícon las hadas deloscuen tos de Hoffman y de Perrault.65

Ello cal deestabiblio tecasedescribecomo "iluminadoytibio" ein cluyeunapintura mural del pintor Carlos Mérida "y en la que Gabriela Mistral relata en verso el cuentodeCaperucita." 66

La citada bibliotecainfantil estuvo anexaalaBiblio tecaMo delo delaSEP que, al igual que la infantil, quedaba instaladahacia fi nales de1923, "en el edi fi cio dela Secretaría de Educación, esquina de la AvenidaRepública Argen tinaylacalledeLuis G onzálezO bregón." 67

Con relaciónalapro ducción deobras, asunto so breelquetam biénin sistieraManriquedeLara, en con tramosqueen 1924laSEP públicóLecturasdásicasparaniños 68

D e esta formalosservi ciosadecuados paraaten deralosniñossevolvían por fin unarealidad.Ademásdelaexisten ciadeunabiblio tecaexprofesoparaestapo blación, la anexaalabiblio tecaModdode la SEP, deeditarlaslec tu rasclá sicas paraniñosyde plan tearquetodabiblio tecapúblicacontaracon unasalainfantil, seanun ciabaquela pro piaBiblio tecaNacionaltendríaunaquesería "el primercen tro infan tildelectura enlaRepública."69Así,seescribió:

LaBiblio tecaNacio nal no permaneceráajenaaestemo vimien to dereno vación.Ultimamentehadado ordenelSeñorMinistro deEducaciónPúbli ca, paraquelacapillaqueocu pó haceañosel departamen to noc turno yque hoy es un simple depósito de libros duplicados y hasta quintuplicados,se transforme en biblioteca infantil. La amplitud del local y la inmejorable

64 "Las bibliotecas públicas en México", p. 138.

65 Ibid

66 Ibid

67 Ibid

68 Lecturas dásicas para niño 1981), 2 vols.

69 "Las bibliotecas Públicas en México", p. 138. 


\section{Investigacón Biblictecoógia v. 16 N o. 33 julio/ diciembre de 2002}

situación del edificio harán de esa biblioteca el primer centro infantil de lectura de la República. ${ }^{70}$

Portan to aun queto davíano helo calizado do cumen tosen losquelaSEPreco nozca explícitamente las aportacio nesdeManriquedeLaraalapo líticabiblio tecaria de Vascon celos, lasfechasdelosescritosaquípresentadosdeMan riquedeLaraylapos terio ridad delasaccio nesan tesmen cio nadasnosllevan ain ferirquesíhubo aporta ción deestabiblio tecariaalapo líticabiblio tecariadeV ascon celosyquelosplanteamientos y sugerencias presentadas por esta autora de una u otra forma fueron escuchadosyquesupropuestasellevóatérmino.

Man riquedeLaraseríareco no cidaporlaSEPporsu destacadaaten ciónen labibliotecapúblicapo pular" AmadaNevo", actuación queconvertiríaaesabiblio tecaen "una delasmejo resymáscon curridasdelaciu dad"71.Eléxi to sedebíaala "buenaad minis tración" que ManriquedeLaralo graraim primirle,yal esfuerzo queestabiblio tecaria pro fesio nal hicieraporpro mo versu uso en trelossecto resdelapo breco munidadala queaten día. Como yasehaseñalado estelo gro sífuereco no cido porlaSEP, queotorgó una comisión con goce de sueldo para que ManriquedeLaraestudiasebiblio teco nomíaen los Estados Uni dos. Poreso cuan do en 1923 sedaco no cerlaapertu radelaBi blioteca Infantil y de la Biblioteca Modelo de laSEP Manrique de Lara se encontraba realizandosusestudiosdebiblioteconomíaenlaciudaddeNuevaY ork.

D esdelosEstadosUnidosManriquedeLaraescribíaeinformabaalasauto ridades no sólo de sus avances escolares en la escuela de bibliotecarios de Nueva Y ork, sino también so breelfun cio namien to delsistemabiblio tecario,ydictabain teresantesleccionesparalosbiblio tecariosso bretemasno vedo soso dein terésparticularparaMéxico.En el primercaso seen con trabael temadelaselección dematerialesparalasbibliotecas, y en el segundo el de catalogación y clasificación, aspecto que venía cobrando importan ciacen tral puesto quelaSEP avanzabaenlaunificación del sistemadeclasifi cación y catalo gación. Esasícomo apartirdelaorganización delaBiblio tecaMo ddo de la Secretaría, se anunciaba que pronto las bibliotecas de la capital y de la república quedaríanorganizadasbajoelmismosistema. ${ }^{72}$

Paralaunificación del sistemadeclasificación, laSEP reconocíahaber enviado a losEstadosUnidosaMaríaLuisaMal do nado "unadelasco labo rado ras [delaSEP] másdiligentes", quien estableció en labiblio tecaMo delo dela SE P "el sistemadetarjetas que existe en la municipalidad de Nueva Y ork." 73 No olvidemos que también ManriquedeLaraseencontrabaestudian do enlaescueladebiblio teco no míaanexaa laBiblio tecaPública NuevaY orkyque, como yasehaseñalado, desdeahíescribíay hacíapro puestasso breel sistemadeclasificaciónyel nivel decatalo gación conmiras aquelaSEPlasadoptara.

70 Ibid

71 "La bibliotecainfantil", p. 202.

72 Ibid

73 Ibid 
Los escritos de Manrique de Lara eran dados a conocer por el D epartamentode Biblio tecasenelboletín Ellibroyd pueblo, y es ahídon de, en marzo de 1923por ejemplo,selepublicabaunartículointitulado precisamente "Laclasificaciónylacatalogacióndelasbiblio tecaspúblicasdeNuevaY ork" ${ }^{74}$ descrip ción conlaqueinstabaalas autoridadesaadoptarenlasbiblio tecaspúblicasmexicanaselsistemaamericano y de alguna manera también a centralizar los procesos técnicos. En esta participación a distancia, relacio nadaconlaclasificación, sehacevisiblelapresenciadeManriquede Lara en laSEP.

Pero al deciresto no sepreten deafirmarqueeraMan riquedeLaraquien descu bría paraMéxico laim portan ciadelaco rrien tebiblio tecariaamericana, puessabemosque estos avances eran co no cidosyad miradosporlosbiblió grafosein telectualesdel Carrancismo, por el propio Vasconcelos y en general por los intelectuales preocupados porlaorganizacióndebiblio tecas, ademásdelosbibliógrafosylosbiblio tecarios.

\section{LA APORTACIÓN DE MANRIQUE DE LARA Y LAFORMACIÓN LECTORA EN MÉXICO YEL MUNDO ACTUAL}

Como sehavisto laprin cipal aportación deMan riquedeLaraen el perio do anali zado seremitealaaten cióneim plan tación deserviciosbiblio tecariosparaniñosyjóvenes, en tan to semillasparacom batirel pro blemadeunaso ciedad no lec to raypropiciareluso delabiblio tecaen México.

Como México continúasien doun paísno lectorelproblemaqueplantearaMan rique deLara, guardan do loscontex tosylasproporcionesdeactualidad, siguesinresolverse.Y lomismo sucedeconlacreacióndebiblio tecasadecuadasparaescolaresyjóvenes, asunto que México no halo grado resolvernien calidad nien can tidad, esto apesardel precio socialquelafaltadeformaciónlecto ratempranaacarreaparanuestrasociedad.

En este orden deco sas, en esen cia, losplan teamien tos, lasideas, loscon cep tosy aún lapro puestadeaten deralapo blación in fan tilyjuvenil con serviciosadecuados apoyadosconelconcurso delaescuelayconbiblio tecariosdebidamentepreparados, siguensiendounproblemadenuestrosdíasenelmedio mexicano.

Lalectu rahasido reco no cidacomo un pro blemaen elám bito mun dial, asílo ubi canauto ridadesdetallain ternacio nal en lamateria, comoJean Hébrard, quien afirma queel fracaso delaedu cación del sigloXX fueunfracaso delalectura. ${ }^{75}$ Enladécada delos ochen talaUNE SCO hacíaunaafirmación queaún hoytienevigen cia: "to davía no existenin gún paísquesesien tasatisfecho conlacan tidady/ o calidad delalectura delamayoríadesushabitantes" .76

74 Juan Manrique de Lara. "La clasificación y la catalogación de las bibliotecas públicas de Nueva York", p. 127

75 [Curso]laformacóndeletoresyescitoresunarevisiónhistónicadidadoparjenHébrardO rganizado porla Embajada de Francia en México y el Fon do deCulturaEco nó micaen octubre, 1999.

76 Ma.TrinidadRo mán, "D esarrollo deunacon ductalecto raatravés delapren dizajesignificativo", p. 27. 


\section{Investigacón Biblictecoógia v. 16 N o. 33 julio/ diciembre de 2002}

A pesar de que el problema de formación de lecto reshamereci do laaten ción de gobiemos y organismosnacionaleseinternacionales, el pro blemadelalecturatiene tal ac tualidad quetan to lasinstitu cio nescomo lospaíses siguen abrien do fo rospara buscarso lu cio nes. Muestradeello esquetenien do como sedenues tro país, en laCiur dad dePueblasecelebraráelVII Con greso Latino americano parael desarro llo dela lecturaylaescrituraenoctubredelpresenteaño(2002).

Todo in dicaquelosargumentospresentadosporMan riquedeLara, haceochenta añosafavordedesarro llarunacon ductalec to rayel gusto porellades delaedad temprana, aun quepu dieran parecerelementalesyobviosfueronvisio narios.Considero que de haberse seguido en nuestro país, constante y sistemáticamente, una política biblio tecaria-educativadirigidaalaniñez,comolo planteabaManriquedeLaradealgunaforma, probablementeelproblemadeunMéxicolectorseríamenor.

Esto no quieredecirqueen México no sehayan em pren dido accio nesparaatenderlapro blemáticadelalectura, en trelascualesfigu ran despuésdelaépo cadeVas con celos, el plan deonceaños de Jai meTo rres Bo det, ini ciado en el go bierno deLópezMateosyquesepro po níaso lu cio narel pro blemadelaedu cación elemen tal. ${ }^{77} \mathrm{El}$ Programa Nacional de Bibliotecas Públicas 1983-1988,78 instaurado durante el periodo de Miguel de la Madrid; el Programa Nacional "Año de la Lectura 19992000 ", ${ }^{79}$ instituido porE rnesto Zedillo,yactualmenteel Pro gramaNacio naldeLectura2001-200680 quehainiciado el go bierno deVicenteFox. No obstan tetodo ello, losplanteamientosdeManriquedeLaraysusideassiguenvigentes.

\section{LANE CE SI DAD DE AVAN ZAR H A CIA UNA SO CIE DAD LEC TO RA: UN PRO- BLEMA SIN RESOLVER EN EL MÉXICO DEL SIGLO XXI}

Comosehaseñalado, uno delospro pó sitosdelMéxico revolucio nario fuecimentarlaso ciedadlecto ramexicanaapartirdelaim plantación deserviciosbiblio tecarios paraniñosyjó venes. Sibien estapro puestaformó parte del ideario del go bierno de Carranza, fueMan riquede Lara quien seapro pió delaim portan ciadeaten deraesta población como base para conformar una sociedad lectora y que recurriera a la bibliotecapararesolversusproblemasalo largo delavida.

Así,en 1921,Juanasepercató dequelapo líticabiblio tecariaem pren didabrillan tementeporVascon celosno contemplaba,almenosex plícitamente,laimplantación de serviciosbiblio tecariosespecíficosparaniñosyjóvenes, asun to quedesdeel pun to de

77 Billy E. Conwart. La dora eucativadeTomesBodkenlonadionalylointernadional,1966. pp.18-23.

78 ElProgramaNacionaldeBibliotecasPúblicas1983-1988ydCentroBibliotecanioNacional:BibliotecaPública deMéxica 1988. 72 p.

79 "Se presen to el Pro gramaNacio nal Año delaLec tu ra 1999-2000" http:/ / www.cnca.gob.mx/ cnca/ nuevo/ diarias/ 310899/ anolectu.html.

80 "Programa de fomento de la lec tu ray el li bro. Haciaun país delec to res" http:/ / www.conaculta.gob.mx/ programa/ fomento. 
vistadeella, debíatenerunlu garprepon deranteen unpaísquerequeríaocu parsede laformaciónlectora.

Parafinalesde1923Vascon celosaten díalafun dación debiblio tecasparaestesectordelapo blación ypoco más tar delapubli cación delas Lecturasparaniños No obstan teelloylasaccio nesto madas porlaspo líticas deLó pez Mateos, delaMadrid,Ze dilloyaho raFox, elpro blemadeformación delecto resydeusuariospermanentesde labiblio tecades demuytem pranaedad siguepresente.Enmiopinión esteim portanteaspecto siguesinseratendidodebidamente.

A pesardequeFelipeG arrido, directordelpro grama "Rinco nesdelalectura" dela SEP, afirmaque "[...]lospro blemasdeunaformaciónlecto raen México aho rason parte de las preocupacionessociales" 81 delosmexicanos, considero quelatrascen den cia delaformación tem pranaenlalecturay delecto resen general, si guesien do sólo parte delaspreo cupacionesdeungrupo redu cido delaso ciedad, prin cipalmentedebibliotecólogos y de algunos otros intelectuales. Ni el problema ha formado parte de las deman dasdelapo blación, nito doslosgo biernoslehan prestado laaten ciónquemerece, detalformaqueel pro blemadeun México lector, señalado porMan riquedeLaraen el go bierno deO bregónsiguesiendounpresen teen estesigloXXI.

Más allá de los esfuerzos discontinuos que en mayor o menor medi dase han he cho en México no sólo por alfabetizar, escolarizar y editar, sino tam bién porfun dar biblio tecas, lascifrasdelpro medio anualdelecturaporhabitan tesiguen sien do bajas. LaCANIEM ${ }^{82}$ señalaquelacifrareal delec tu radel pueblo mexicano es demenosde un libro anual por mexicano, dicha cifra se aleja grandemente de los cuatro libros anualesporhabitan tequeseñalalaUNESCO como mínimo acep tableparaconsi de raraun país como lector,83yeso sinmen cio naraquílacalidad delo leído.

O tro in dicadordelapo brezadepo sibilidadesdelecturaparanuestrapo blaciónse puedeobtenerdel to tal delibrosdein terésgeneral quesegún datosdelaCANIEMse pro dujo en 1999. Ladivisión deestedato en treel to tal depo blación, deacuerdo con el cen so del 2000, in dicaqueacadahabitan teleco rrespon deríaun .47 delibro, delos clasificadoscomo deinterésgeneral. 84

Concretándonos a las posibilidades de lecturadel sectordondepo dríacultivarse unapo blaciónlecto ra, niñosyjóvenes, losin dicado resqueheobtenidoyqueexisten actualmentesonmuydesalentadoreshelosaquí:

81 "Tres libros por mexicano", El infomadar diario indqpendiente, Guadalajara, Jal., México, (5 julio, 1999). http:/ / www.informador.com.mx/ Lastest/ Jul99/ 05AR10A.htm.

82 "En México seleemenos deun libro porperso naalaño:CANIEM", BdeínUNAMDGCS-0402,(4 mayo, 2002). http:/ / www.dgi.unam.mx/ boletín/ 2002 0402.html.

83 Ilid

84 CámaradelaIndustriaEdito rial Mexicana, “Actividad edito rial 1998-1999”, 2001..p. 20. y “Población total por grandes grupos de edad según entidad federativa, 2000". http:/ / www.inegi.gob.mx/ estadistica/ espanol/ sociodem/ sexo/ sex_02.html. Los datos seto maron delas obras an terio resysonelresultado dedividirlosejem plaresqueen 1999sepro dujeron delibrosdein terés general $(46,753,932)$ entre la población total del cen so de2000 $(97,483,412)$ habitan tes. 
2 libros de texto para la población de 5 a 14 años, ${ }^{85}$

* 0.67 libros infantiles para la población hasta de 9 años, 86

* 0.12 libros juveniles para la población de 10 a 14 años y87

* 0.48 libros infantiles y juveniles para la población de hasta 14 años.88

Silasoportunidades deun mayorespectro delecturaladan lasbiblio tecas, consi dero quelaaten ción tem pranaenbiblio tecasparacoadyuvaralaformacióndelectoressiguesien do, como sesostienealo largo deestedo cumen to, unasun to vigen teen nuestrasociedad.

Enunpaíscomo el nuestro, con escaso pro medio delectura, queen general puede clasificarsecomo no lectoryen don dealmenosun 30\% delapo blación esdeniñosy jó venes, muy pocaim portan ciaselehadado alaaten ción deestepro blemadesdesu base,laniñez.Eldesarro llo biblio tecario paraestapo blación guardarelación directa conlaverdaderaconstitu ciónyfuncionamien to delasbiblio tecasesco lares, infantilesyjuveniles.

Lapo blacióninfantilyjuveniltanto delMéxico de1921 como delde2002representabayrepresentamásdel 30\% delapo blación to tal.D eacuerdo con el cen so depo bla ción de 1921, deun to tal de 14, 334780 habitan tes5, 505503 eran meno res dequin ce años, cifraquerepresentabaun $38.4 \%$ delapo blaciónto tal. 89 En el cen so del año 2000 las cifras relativas no han variado considerablemente y hoy, casi igual que hace 80 años, seen cuen traqueel $33.4 \%$ delapo blación tienemenosde15años(32, 586, 973 habitantes). ${ }^{90} \mathrm{D}$ e ahí que la esperanza de cambiar de un país no lector a uno lector

85 CámaradelaIndustriaEditorialMexicana, “Actividadeditorial1998-1999”, 2001. p. 20. y "Po bla ción to tal porgran des gru pos deedad se gún en ti dad fe derativa, 2000".

http:/ / www.inegi.gob.mx/ estadistica/ espanol/ sociodem/ sexo/ sex_02.html. Los datos se to maron de las obras anteriores y son el resultado de dividir $(44,43 \overline{4}, 190)$ de libros texto entre $(21,951,816)$ millo nes depo bla ción 5 a 14 años.

86 CámaradelaIndustriaE ditorialMexicana, “Actividadedito rial 1998-1999”,2001.p.23. y “Po bla ción to tal porgran desgru pos deedad según en ti dad federativa, 2000".

http:/ / www.inegi.gob.mx/ estadistica/ espanol/ sociodem/ sexo/ sex_02.html. Los datos se to maron de las obras anteriores y son el resultado de dividir Libros infantiles $(14,671,817)$ entre $(21,850,480)$ mi llo nes de po bla ción de 0 a 9 año.

87 CámaradelaIndustriaE ditorial Mexicana, “Actividadedito rial 1998-1999”, 2001.p.23. y“Po bla ción to tal porgran desgru pos deedad según en ti dad federativa, 2000".

http:/ / www.inegi.gob.mx/ estadistica/ espanol/ sociodem/ sexo/ sex_02.html. Los datos se to maron de las obras anteriores y son el resultado de dividir $(1,25 \overline{4}, 493)$ libros juveniles entre $(10,736,493)$ depo blación de 10 a 14 años.

88 CámaradelaIndustriaE ditorialMexicana, "Actividadedito rial 1998-1999", 2001.p.23. y "Po bla ción total por grandes grupos de edad según entidad federativa, 2000". http:/ / www.inegi.gob.mx/ estadistica/ espanol/ sociodem/ sexo/ sex 02.html.Los da tos seto maron delas obras an terio resyson el resultado dedividir $(15,926,310)$ lib̆rosinfan tilesyjuvenilesen tre $(32,586,973)$ de población de 0 a 14 años.

89 "Población total por grandes grupos de edad y relación de dependencia, 1895-1990" http:/ / dnsI.mor.itesm.mx/ DIV_ACADEM/ DEMS/ est/ pob04.html

90 "Po blación to tal porgran desgru posdeedad según en ti dad federativa, 2000". http:/ / www.inegi.gob.mx/ estadistica/ espanol/ sociodem/ sexo/sex_02.html. 
pudieraes tar en laaten ción aestapo blación yasu seno familiar. Pero veamoscomo estáhoyelpano ramadebibliotecasparalosniñosyelpúblicoengeneral

No obs tan te quelaEstadísticabásicaddsistemanacional debibliotecas, 1995señalaque existen en el país 4,13391 bibliotecas esco lares, dichacifradebein cluirlaspequeñas co leccio nescon quecuen tanlasescuelas, queenmu chasocasionesson resguardadas tan ab surdaycelo samen teque casino seprestanniapro feso resniaalum nos. Pero aun aceptando que la cifra de 4,133 representara verdaderas bibliotecas escolares, esta cantidad sería insuficiente con relación a la población infantil y juvenil mexica na, ${ }^{92}$ pues in dicaríaquecadabiblio tecaestaríaaten dien do po ten cialmenteamásde 7000niñosyjóvenes, lacualesunaciframuyelevada. Sialasanterio resbiblio tecas les sumamoselto tal debiblio tecaspúblicas(5,490en 1995),93 con si deran do queen ge neral han devenido en apoyo paraesco lares, elpro medio deaten ción porbiblio tecaa niñosyjó venesmejo raría, pero seguiríasien do elevado, serían un poco másde 3000 losniñosyjóvenesaten didos.Estan graveel pro blemadedesarro llo bibliotecario en México quelacifraquesuman estosdostiposdebiblio tecas(lasesco laresylaspúblicas),representamásdel $87 \%$ delescaso desarrollobibliotecario delpaís.

Aunsien do conscien tesdequeel pro blemadeunaso ciedadlecto raesun fenó me no dealtacom plejidadydequelasbiblio tecasengeneralyaunlasdestinadasaniños y jó venesporsisolasno represen tan laso lu ción, laim plan taciónylaoperativi dad de biblio tecassiguensien do consideradas, como elementosimportantesquefo menta rían elestablecimien to deunaso ciedadlectora.JesúsG alera, vicepresidentedela CANIEM, por ejemplo, recomienda en mayo de este 2002, que un aspecto importante paraaumentarlosín dicesdelecturasería "lacreación debiblio tecasen barriosycoloniasypo blaciones." 94

En con cordan ciacon elúl timo au torcitado y con lapro piaMan ri quedeLarase considera que las bibliotecas escolares y las denominadas infantiles desempeñarían unpapelim portanteen elciclo deformacióndelasnuevasgeneracionesparaavanzar hacia un país de lectores que aprendan a servirse de las bibliotecas a lo largo de su vida. Manrique de Lara sigue siendo parte de esta historia en la que ha habido momentos, go biernosyperso nasquesehan ocupado delabúsquedadesolucio nesypor eso hoysetraenacuentalosargumentos, planteamientos, proyectosyestrategiasque ellahicieraaesterespecto.

91 SecretariadeEducaciónPública.Dirección G eneraldePlaneación,Pro gramaciónyP resupuesto. Estadísticasbásicas da SistemaNacional deBibliotecas, 1995. p. 10

92 "Población total por sexo según grupos quinquenales de edad, 2000" http:/ / www.inegi.gob.mx/ estadistica/ espanol/sociodem/ sexo/ sex_02.html.

93 SecretariadeEducaciónPública.Dirección G eneraldePlaneación,Pro gramaciónyP resupuesto. Estadísticasbásicas de SistemaNacional deBibliotecas, 1995. p. 10

94 "En Méxi co selee menos de un li bro porperso naal año: CANIEM", BdeínUNAMDGCS0402, (4 mayo, 2002) http:/ / www.dgi.unam.mx/ boletín/ 2002 0402.html 


\section{CONCLUSIONES}

En virtud de que México no ha logrado que la lectura se constituya en un hábito que caracterice a nuestra sociedad, consideramos que en esencia la propuesta de Manrique de Lara de cimentar la formación lectora y de usar la biblioteca para instruirse, do cumen tarsey distraerse, apartirdelaaten ción biblio tecariaalapo blación infantilyjuvenil,éstesiguesien do un pun to dereferen ciaim portanteparalosen cargadosdeencauzarlapolíticabibliotecarianacional.

Consideramosquelapérdidadederro tero enlafundaciónyconso lidación deverdaderosserviciosbiblio tecariosparalapo blacióninfantilyjuvenil,conlaconsecuenteformación debiblio tecólo gosparaaten derespecialmenteaestapo blación deprofesores que promuevan el uso de la información, el impulso de la producción de la literatura conveniente que coadyuve al desarro llo dehabilidadesinformativasy lecto ras, pro pues tos hace ochen taaños porMan riquedeLara, hasido uno delosfac toresquehan marcado el atraso en relación con el uso delain formaciónylaprácticade lalec tu raen queto davíaseen cuen tranuestro país. Porotrapartehabríaqueagre gar queesnecesarialado tación delasnuevastec no lo gíasdelainformaciónylapreparaciónparasuuso desdelamástiernaedad, en escuelasybiblio tecas.

\section{BIBLIOG RAFÍA CON SULTADA}

Arias Bernal, María. “Las biblio tecaspúblicasen losEstados Unidos:informe presentado al Subsecretario de Instrucción Pública y Bellas Artes, en marzo de 1915." En Bdéín deeedraión: órgamodela Seretańa de Instrución Púdicay Bellas Artes, t. I, n. 2, nov. 1915 : 109-113.

"Labiblio tecainfantil." En Ellibroyd pueblo, t. II, n. 8-10, oct./ dic. 1923 : 202.

"Las bibliotecas públicas en México." En El libroyd puddo, t. II, n. 6-7, ago./ sep. 1923 : 137-139.

Cámara de la Industria Editorial Mexicana, Adividad eitarial 1998-1999. - México : CANIEM, 2001.- 71 p.

Conwart, Billy E. LadbraeducativadeTonesBodktenlonacionalylointernadi nal. - México:ElColegio deMéxico,1966. - 53p.- (Jornadas:59).

[Curso] la formaión deletoresy escitores: una reisión históica didadoporJæn Hearard O rganizado porlaE m bajadadeFran cia, en México yel Fondo de Cultura Económica en octubre de 1999.

"D eclaracionesdelseñorlicen ciado donJoséVasconceloscon motivo de la toma de posesión del cargo de rec tordelaUniversi dad Nacio nalde México." En BdeéndelaUnivesidad, t. I, n. 1, ago. 1920 : 7-13. 
"Institucióndebibliotecasinfantilesenlasescuelasprimariassuperiores." En Bdeín deeeduracoón: órgamo dela Sertaria deInstrucción PúdicayBdlas Artes, t. I, n. 3, feb. 1916 : 33-34.

"Jira [sic] cultural de maestros por algunas de los Centros escolares más im portan tesdelosEstados Unidos." En Bdeéndeeducacoón:ớganodla Seertanáa deInstrucción Pública y BdlasA Ates, t. I, n. 2, nov. 1916 : 72.

"LabordelCiudadano D.Venustiano Carranza, encargado delPo derEje cutivo del Gobierno Constitucionalista de México, en la Secretaría de Instruc ción PúblicayBellasArtes, duran telagestión del Ingeniero Fé lix F.Palavicini." En Bdeíndæeducaaón: órganodelaSecetaniadeInstrucción PúdlicayBellas Artes, t. I, n. 2, nov. 1915 : 223-227.

Lechras dásicas para niños - México : SEP, D epartamento Editorial, 1981. $-2 \mathrm{v}$.

Manrique de Lara, Juana. "La biblioteca pública y los alumnos de las escuelas preparatorias." En Billos, t. III, n. 145, oct. 29, 1921 : 175.

- - -. "Las bibliotecas infantiles y juveniles." En Biblos t. IV, n. 158, 159, ene, 28; feb. 4, 1922. : 15, 18-19.

- - -. "Las bibliotecaspúblicas y los alumnos de las escuelas primarias." En Bildos t. III, n. 146, nov. 5, 1921 : 179.

- - -. "La clasificación y la catalogación de las bibliotecas públicas de Nueva Y ork." En El libroyd puddo, t. II, n. 4-5, mar. 1923 : 127.

MoralesCampos, Estela. Eduraaónbibidtedoóǵcaen México1915- 1954.México :UNAM,Cen troUniversitario deInvestigacionesBibliotecológicas, 1988. - $112 \mathrm{p}$.

[“Primer editorial de Biblos”]. En Bildos t. I, n. 1, ene. 18, 1919 : 1.

El Programa Naaional deBiblicteras Públicas 1983-1988ye CentroBibliotecanio Nacional:BibliotecaPúblicadeMéxica - México :SEP,D irección G eneral de Bibliotecas, 1988. - $72 \mathrm{p}$.

Q uintana Pali, G uadalupe. Lasbiblicteas públicas en México 1910-1940. México : SEP, Dirección G eneral de Bibliotecas, 1988. - 485 p.

"Reglamento de las bibliotecas públicas." En El libroyd puddo t. I, n. 4, jun. 1922 : 26. Alcalce:J.Vascon celos.ElSecretario deE ducaciónPú blica.

Ro mán Haza, Ma.Trinidad. "D esarrollo deunacon ductalec to raatravés del aprendizaje significativo." En Investigaaón biblidteedógica : archivono máa, biblictedooǵa einformacón, t. III, n. 6, ene./ jun. 1989 : 27-29.

Sametz de Walerstein,Linda. Vasconcelosdhombreddlibra laépocadbarode lasdibliotecas - México :UNAM,Instituto deInvestigacionesBibliográficas, 1991. $-227 \mathrm{p}$. 
"ElSeñorD on Venustiano Carranzaesno solamenteelamigo delpueblo, sino, en parti cu lar, delosniñosdeescueladeMéxi co." EnBolet́ndeedt cacón : órgamo dela Serzania deInstrucción Pública y Bdlas Artes, t. I, n. 2, nov. 1915 : 101-108.

SecretaríadeEdu cación Pública. Dirección GeneraldePlaneación. ProgramaciónyPresupuesto. EstadísticasbásicasddSistemaNacionaldeBiblio teras-México : SEP,Direc ción G eneral dePlaneación,Pro gramacióny Presupuesto, 1982. - $263 \mathrm{p}$.

Vasconcelos, José. "Conferencia leída en el 'Continental Memorial Hall' de Washington." En Bdéíndela SecretanadeEducacónPública, t. I, n. 3, ene. $1923: 5-16$.

\section{PÁgINAS WEB}

"En México seleemenosdeun libro porperso naal año:CANIEM" Bdeín UNAM-DGCS-0402, (4 mayo, 2002).

http:/ / www.dgi.unam.mx/ boletín/ 2002_0402.html [consultado el 25 de junio de 2002

"Pro gramadefo men to delalec tu rayel libro. Haciaun paísdelecto res". http:/ / www.conaculta.gob.mx/ programa/ fomento [consultado el 9 de diciembre de 2002]

"Se presentó el Programa Nacional Año de la Lectura 1999-2000". http:/ / www.cnca.gob.mx/ cnca/ nuevo/ diarias/ 310899/ anolectu. $\mathrm{html}$ [consultado el 10 de diciembre de 2002]

"Treslibrospormexicano", Elinformadardiarioindependiente,G uadalajara, Jal., México, (5 julio, 1999). http:/ / www.informador.com.mx/ Lastest/ Jul99/ 05AR10A.htm [consultado el 25 de junio de 2002].

"Población total por grandes grupos de edad según entidad federativa 2000"

http:/ / www.inegi.gob.mx/ estadistica/ espanol/ sociodem/ sexo/ sex 02.html [consultado el 25 de junio de 2002]

"Población total por grandes grupos de edad y relación de dependencia, 1895-1990"

http:/ / dnsI.mor.itesm.mx/ D IV_ACAD EM/ DEMS/ est/ pob04.html. [consultado el 25 de junio de 2002]

"Población total por sexo según grupos quinquenales de edad, 2000" http:/ / www.inegi.gob.mx/ estadistica/ espanol/ sociodem/ sexo/ sex 01.html

[consultado el 25 de junio de 2002] 


\section{DOCUMENTOS DE ARCHIVO}

AHBN, Fondo Administrativo. Carpeta 77. Exp. 1908 No. 2870. [Relación de oficios emitidos por la Biblioteca Nacional durante el año de 1917].

AHBN,Fondo Administrativo.Carpeta88.Exp.2355.No.3324.[Recorte delartículo "Biblio tecasInfantiles" porBeatriz G alindo, publicado en elperiódico El Solde Madrid.Esteartículo fueen viado porel Rectora la Biblioteca Nacional, 28 de enero de 1919].

AHBN, Fondo delaEscuelaNacionaldeBiblio tecariosyArchivistas.CaF peta 114. Exp. 3360. No. 4354. [Sugerencia del director de la Escuela Nacional de Bibliotecarios y Archivistas al Ministro de Educación, de in cluir en los pro gramas de Len gua Na cio nal el tema del manejo debi bliotecas y uso, selección y prácticas de asimilización de libros. 20 de abril de 1916].

A HBN,Fon do delaEscuelaNacional deBiblio tecariosyArchiveros.Car peta115.Exp.3488.No.4490. [O ficio turnado porLuis Manuel Rojas, alSubdirectordelaBiblio tecaNacio nalyen cargado deEscuelaNacional de Bibliotecarios y Archiveros solicitándole se elabore una lista de obrasen es pañol con venien tesparaniños meno res de15añosparasa tisfacer la petición de la Srita. Leonore Power, Children's Librarian. 9 de agosto de 1917].

AHSEP, Fon do deConcen tracióndelaDirección GeneraldeAdministra ción Escolar. Exp. D / 131/ 4949. D ocumentos de Juana Manrique de Lara. [Co piadelcertificado quehaceconstarquelaseño ritaJuanaManriquecursó estu dios, en tre1911y1913en el Instituto Normal Meto dis ta, Ex pedido en PuebladeZara go za, alos seis días del mes dejunio de 1935]. 